\title{
A POSSIBILITY FOR USING AN APPLE UNDULATOR TO GENERATE A PHOTON BEAM WITH TRANSVERSE OPTICAL MODES
}

\author{
Shigemi Sasaki", Ian McNulty, ANL, Argonne, IL 60439, U.S.A. \\ Taihei Shimada, JAEA, Tokai, Ibaraki, Japan
}

Abstract

We investigate use of an APPLE-type undulator [1] for generating Laguerre-Gaussian (LG) and HermiteGaussian (HG) mode beams. We find that the second harmonic radiation in the circular mode corresponds to an LG beam with $l=1$, and the second harmonic in the linear mode corresponds to an $\mathrm{HG}$ beam with $l=1$. The combination of an APPLE undulator and conventional monochromator optics may provide an opportunity for a new type of experimental research in the synchrotron radiation community.

\section{INTRODUCTION}

Photons that carry orbital angular momentum are of great interest to the optics and laser communities [2]. This exotic property of photon beams was recently demonstrated in the x-ray regime [3] and may be useful to probe angular momentum in matter [4]. However, by comparison to the visible-light regime, it is difficult to fabricate efficient achromatic optics to generate these optical modes in x-rays. In spite of these inconveniences, there has been no investigation of the possibility of using a synchrotron light source to directly generate an x-ray beam with transverse optical modes. As is known very well, linear undulator does not generate even harmonics on axis and a helical undulator does not generate any higher harmonics on axis. Due to this fact, off-axis higher harmonic radiations (especially even harmonics) have been considered useless, and their properties have not been investigated intensively.

In this paper, we demonstrate that the higher harmonic radiation from an APPLE-type undulator carries orbital angular momentum in the circular polarization mode, and carries the state of Hermite-Gaussion (HG) mode or Transverse Electro-Magnetic (TEM) mode in linear polarization mode.

\section{EQUATIONS OF RADIATION}

In any operation modes (linear, elliptical, or circular) of an APPLE undulator, which is originally proposed, the phase difference between the horizontal and the vertical components of the periodic magnetic field is $\pi / 2$, and hence the axis of the polarization ellipse stays in the horizontal plane. For this case, components of the radiation amplitude in the horizontal, $\mathrm{x}$, and vertical, $\mathrm{y}$, directions are given by the following equations [5]:

$$
\begin{aligned}
& A_{x}=2 \gamma \theta \cos \phi S_{0}-K_{y}\left(S_{1}+S_{-1}\right) \\
& A_{y}=2 \gamma \theta \sin \phi S_{0}+i K_{x}\left(S_{1}-S_{-1}\right)
\end{aligned}
$$

where

$$
S_{q}=\sum_{p=-\infty}^{\infty} J_{p}(Y) J_{n+2 p+q}(X) e^{i(n+2 p+q) \Phi},
$$

and

$$
\begin{aligned}
& X=2 n \xi \gamma \theta \sqrt{K_{y}^{2} \cos ^{2} \phi+K_{x}^{2} \sin ^{2} \phi}, \\
& Y=n \xi\left(K_{y}^{2}-K_{x}^{2}\right) / 4, \tan \Phi=\left(K_{x} / K_{y}\right) \tan \phi, \\
& \xi=1 /\left(1+\gamma^{2} \theta^{2}+K_{x}^{2} / 2+K_{y}^{2} / 2\right) .
\end{aligned}
$$

In the equations above, $\theta$ is the observation angle from the radiation axis; $\phi$ is the azimuthal angle in the $\mathrm{x}-\mathrm{y}$ plane at an observer's position; $n$ is the harmonic number; $q=-1,0,1$; and $J_{\mathrm{n}}$ is the $n$-th order Bessel function.

For pure circular polarization mode, $\mathrm{K}_{\mathrm{y}}=\mathrm{K}_{\mathrm{x}}=\mathrm{K}$ and $X=2 n \xi \gamma \theta K, \mathrm{Y}=0, \Phi=\phi$. Then, after a simple manipulation, we get:

$A_{x}=e^{\mathrm{i} n \phi}\left\{2 \gamma \theta \cos \phi J_{n}(X)-K\left(J_{n+1}(X) e^{\mathrm{i} \phi}+J_{n-1}(X) e^{-\mathrm{i} \phi}\right)\right\}$

$A_{y}=e^{\mathrm{i} n \phi}\left\{2 \gamma \theta \sin \phi J_{n}(X)-\mathrm{i} K\left(J_{n+1}(X) e^{\mathrm{i} \phi}-J_{n-1}(X) e^{-\mathrm{i} \phi}\right)\right\}$.

For the linear mode, $K_{\mathrm{x}}=0$ and $\Phi=0$, we get

$A_{x}=2 \gamma \theta \cos \phi S_{0}-K_{y}\left(S_{1}+S_{-1}\right)$,

$A_{y}=2 \gamma \theta \sin \phi S_{0}$.

Again, after additional manipulation, we get:

$$
\begin{aligned}
& A_{x}=2 \gamma \theta \cos \phi S_{0}-\frac{1}{\xi \gamma \theta \cos \phi}\left(S_{0}+\frac{2}{n} S_{2}\right), \\
& A_{y}=2 \gamma \theta \sin \phi S_{0},
\end{aligned}
$$

where:

$$
S_{2}=\sum_{p=-\infty}^{\infty} p J_{p}(Y) J_{n+2 p}(X) .
$$

Equations (2) and (3) are valid for any harmonics of radiation for the circular mode and linear mode, respectively.

\section{PROPERTIES OF THE SECOND HARMONIC}

The total intensity distribution is obtained by adding the absolute square of the horizontal component, $\left|\mathrm{A}_{\mathrm{x}}{ }^{2}\right|$, and that of the vertical component, $\left|\mathrm{A}_{\mathrm{y}}{ }^{2}\right|$.

Figure 1 shows the calculated angular distributions of the radiation intensity in the circular mode for the fundamental and second harmonic frequencies for a single electron. The intensity distribution has radial symmetry, and the second harmonic has the so called "optical donut"

\#sasaki@aps.anl.gov 
shape associated with Laguerre-Gaussian-mode beams carrying orbital angular momentum (OAM) [6]. However, this shape does not necessarily mean the radiation has the property of OAM.

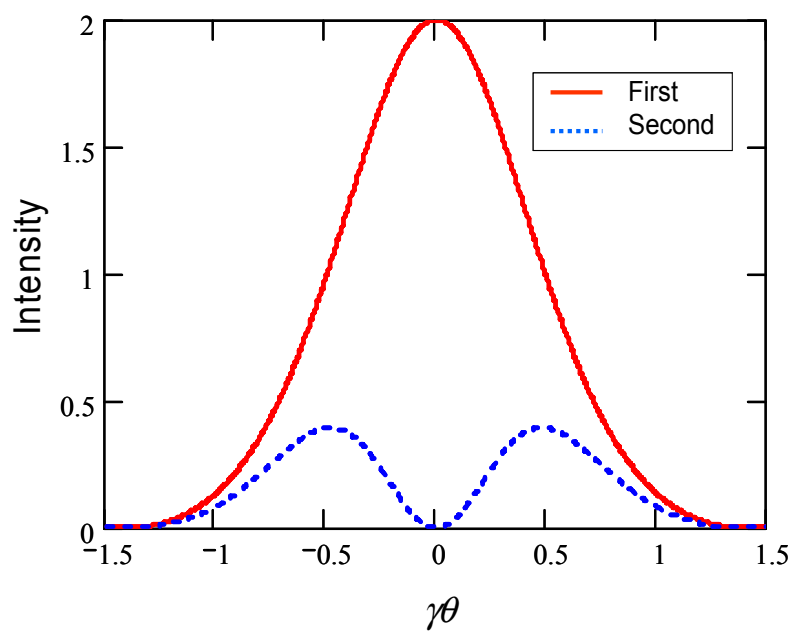

Figure 1: Angular intensity distributions of fundamental (solid line) and second (broken line) harmonics. The deflection parameter $K$ equals one.

Table 1 shows the relation between the azimuthal angle and the phase of the electric field of the radiation. It is clearly shown that the sign of amplitude of the electric field is in the opposite position with respect to the radiation axis (compare $\phi=0$ and $\phi=\pi$, or $\phi=\pi / 2$ and $\phi=2 \pi / 3$ in each amplitude component). This means that the phase of radiation shifts 180 degrees by the 180-degree rotation of the azimuthal angle around the axis.

Table 1: Azimuthal Angle, $\phi$, Dependence of Amplitude

\begin{tabular}{|c|c|c|}
\hline$\phi$ & $A_{\mathrm{x}}$ & $A_{\mathrm{y}}$ \\
\hline 0 & $(2 \gamma \theta-4 K / X) J_{2}(X)$ & $-2 \mathrm{i} K J_{2}{ }^{\prime}(X)$ \\
\hline$\pi / 2$ & $-2 \mathrm{i} K J_{2}{ }^{\prime}(X)$ & $-(2 \gamma \theta-4 K / X) J_{2}(X)$ \\
\hline$\pi$ & $-(2 \gamma \theta-4 K / X) J_{2}(X)$ & $2 \mathrm{i} K J_{2}{ }^{\prime}(X)$ \\
\hline $3 \pi / 2$ & $2 \mathrm{i} K J_{2}{ }^{\prime}(X)$ & $(2 \gamma \theta-4 K / X) J_{2}(X)$ \\
\hline
\end{tabular}

Figure 2 shows the time dependence of the electric field for the second harmonic for the zero-emittance case computed by the code UR [7].

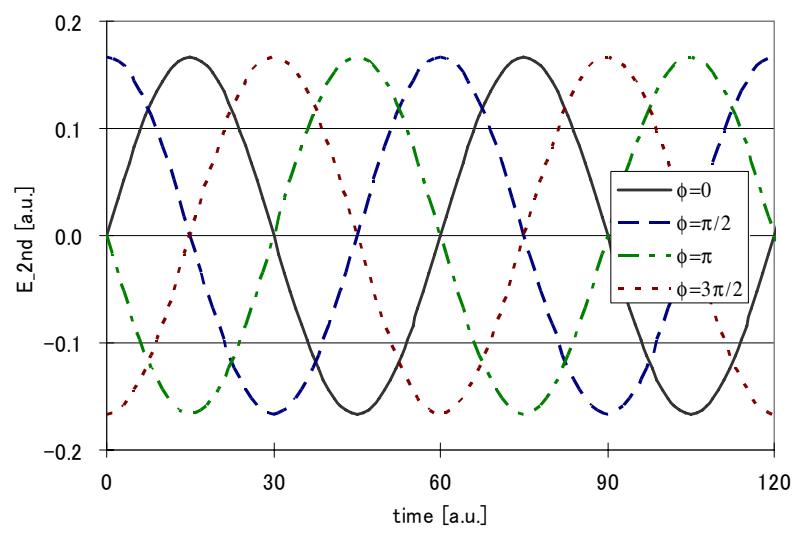

Figure 2: Time dependence of electric field for secondharmonic wavelength.

The phase information for the second harmonic can be retrieved by Fourier decomposition of the time dependence of the electric field. In this figure, as the azimuthal angle rotates by $\pi / 2$, the peak amplitude shifts by $\lambda / 4$. This helical phase signature is evidence that the second harmonic carries OAM with an azimuthal mode index of $l=1$, where $l$ is the azimuthal quantum number.

In contrast with radiation in the circular mode, radiation in the linear mode does not carry OAM. Instead, it may have TEM mode, which is equivalent to the HG mode. Figure 3 shows the angular intensity distribution of the $\sigma$-mode (horizontal linear polarization component) of second harmonic radiation in the linear mode at $K=1$.

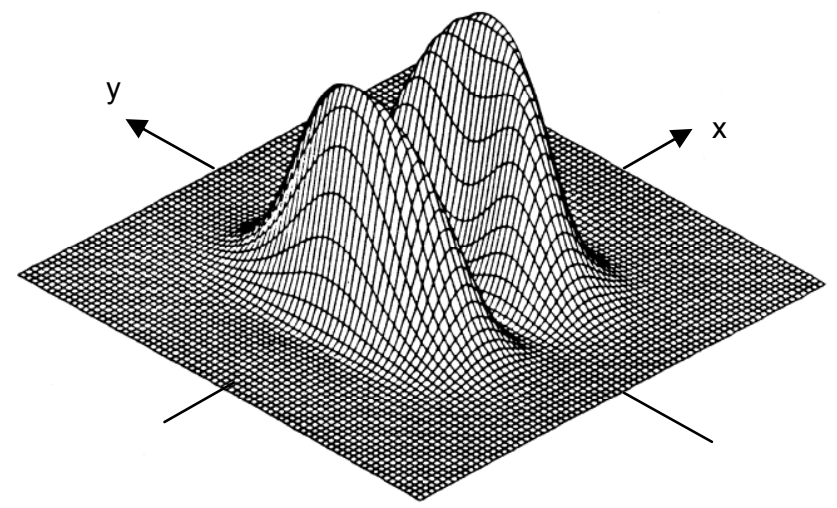

Figure 3: Angular intensity distributions of the $\sigma$ component of the second harmonic in the linear mode at $K=1$.

By putting $\mathrm{n}=2$ and $\phi=0, \pi$, and from the fact that $\mathrm{S}_{0}(\mathrm{X})=\mathrm{S}_{0}(-\mathrm{X})$ and $\mathrm{S}_{2}(\mathrm{X})=\mathrm{S}_{2}(-\mathrm{X})$ for $\mathrm{n}=$ even number, we get:

$$
A_{\mathrm{x}}(\text { for } \phi=\pi)=-A_{\mathrm{x}}(\text { for } \phi=0) .
$$

This means that the phase of radiation amplitude at $\phi=0$ and $\phi=\pi$ is opposite $\left(180^{\circ}\right.$ difference) from each other, 
which may correspond to the $\mathrm{HG}$ mode with $l=1$ or equivalent to the $\mathrm{TEM}_{01}$ mode.

\section{CONCLUSION}

We investigated the properties of higher harmonic radiation from an APPLE-type undulator. We found that the second harmonic not only carries spin (circular polarization) but also the orbital angular momentum in the circular mode, and in the linear mode it carries the TEM mode. This new radiation property can be useful for various kinds of synchrotron radiation users' experiments.

\section{ACKNOWLEDGMENTS}

We thank Professor Padgett for insightful comments during an intensive discussion with us.
This work is supported by the U.S. Department of Energy, Office of Science, Office of Basic Energy Sciences, under Contract No. DE-AC02-06CH11357.

\section{REFERENCES}

[1] S. Sasaki, Nucl. Instrum. Methods A 347, 83, (1994).

[2] M. Padgett, J Courtial, L Allen, Physics Today, p. 35, May, 2004.

[3] A. G. Peele et.al., Optics Letters, 27, 1752 (2002).

[4] M. VanVeenendaal and I. McNulty, Phys. Rev. Lett. 98, 157401 (2007).

[5] R. P. Walker, Proceedings of CERN Accelerator School, CERN 98-04, p.129.

[6] A. G. Peele et al., Optics Letters 27, 1752 (2002).

[7] R. J. Dejus, A. Luccio, Nucl. Instrum. Methods A 347, 61 (1994). 